\title{
Optical polarimetric observations of the microquasar LS 5039^
}

\author{
J. A. Combi ${ }^{1,2}$, S. A. Cellone ${ }^{3}$, J. Martí ${ }^{1}$, M. Ribó ${ }^{4}$, I. F. Mirabel ${ }^{4,5}$, and J. Casares ${ }^{6}$ \\ 1 Departamento de Física, Escuela Politécnica Superior, Universidad de Jaén, Virgen de la Cabeza 2, 23071 Jaén, Spain \\ e-mail: [jcombi;jmarti]@ujaen.es \\ 2 Instituto Argentino de Radioastronomía, C.C.5, (1894) Villa Elisa, Buenos Aires, Argentina \\ 3 Facultad de Ciencias Astronómicas y Geofísicas UNLP, Paseo del Bosque, B1900FWA La Plata, Argentina \\ e-mail: scellone@fcaglp.fcaglp.unlp.edu.ar \\ 4 Service d'Astrophysique, CEA Saclay, Bât. 709, L'Orme des Merisiers, 91191 Gif-sur-Yvette Cedex, France \\ e-mail: [mribo;mirabel]@discovery.saclay.cea.fr \\ 5 Instituto de Astronomía y Física del Espacio, CONICET, C.C.67, Suc. 28, 1428 Buenos Aires, Argentina \\ ${ }^{6}$ Instituto de Astrofísica de Canarias, 38200 La Laguna, Tenerife, Spain \\ e-mail: jcv@1l.iac.es
}

Received 8 June 2004 / Accepted 4 August 2004

\begin{abstract}
We present the first optical polarimetric observations of the runaway microquasar LS 5039. Our results reveal the presence of a large amount ( $\sim 5 \%)$ of polarized emission towards this binary system. By combining polarimetric and spectroscopic observations of some stars in the field together with available statistical information on the galactic interstellar polarization of the region, we have estimated and subtracted the contribution of the interstellar polarization in this direction. As a result, we obtain an intrinsic polarization of $\sim 3 \%$ for the object, much higher than what would be expected from jet emission in the optical domain. We suggest that the polarized light originates by electron Thomson scattering in the stellar envelope of the companion star. This allows us to constrain the size of the region where the polarized emission originates, as well as estimating the scattering electronic density and the wind velocity at such distance.
\end{abstract}

Key words. stars: individual: LS 5039 - X-rays: binaries - stars: binaries: general - polarization

\section{Introduction}

The high mass X-ray binary system LS 5039 is one of the about sixteen confirmed Galactic microquasars (Paredes et al. 2002; Ribó 2002). Recent astrometric studies carried out by Ribó et al. (2002), show that it is a runaway system escaping from the Galactic plane with a systemic velocity of $\sim 150 \mathrm{~km} \mathrm{~s}^{-1}$.

LS 5039 is a bright $V \sim 11.2$ star with an $\mathrm{ON6.5 \textrm {V } ( ( \mathrm { f } ) )}$ spectral type (McSwain et al. 2004), located at a distance of $2.9 \pm 0.3 \mathrm{kpc}$ (Ribó et al. 2002). The binary system has a short orbital period of $P_{\text {orb }}=4.4267 \pm 0.0005$ days and a high eccentricity of $e=0.48 \pm 0.06$ (McSwain et al. 2004). At radio frequencies, the source has a non-thermal spectral index with moderate, but not periodic, variability. No strong radio outbursts have been ever detected (Martí et al. 1998; Ribó et al. 1999; Ribó 2002).

In X-rays, the source presents a hard spectrum up to $30 \mathrm{keV}$, but no pulsed nor periodic emission has been detected (Ribó et al. 1999; Reig et al. 2003). The possibility that LS 5039 is a $\gamma$-ray emitter was suggested by Paredes et al. (2000),

* Based on observations made at the Complejo Astronómico El Leoncito, which is operated under agreement between CONICET and the National Universities of La Plata, Córdoba, and San Juan. who proposed its association with the unidentified $\gamma$-ray source 3EG J1824-1514 (Hartman et al. 1999). In this context, the $\gamma$-rays would be produced by inverse Compton upscattering of lower-energy photons by the non-thermal relativistic $\mathrm{e}^{-}$population responsible of the radio emission. Based on wind accretion models McSwain et al. (2004) suggest a neutron star as the compact object in the binary system, which would have an inclination angle between $40^{\circ}$ and $60^{\circ}$.

Although several physical parameters of the system are known, there is not yet a clear picture allowing to explain the broadband emission detected from it. Therefore, new observations that help to constrain the properties of the system are needed.

Optical polarimetric observations are a useful tool in the study of intrinsic polarization of X-ray binaries, since they can give us important information about their physical and geometrical properties. This kind of studies have been carried out by Scaltriti et al. (1997) for the microquasar GRO J1655-40, where they found a significant amount of intrinsic linear polarization ( 3\%) in the VRI bands, with the polarization direction being parallel to the accretion disk plane. In a following paper on this object, Gliozzi et al. (1998) detected oscillations in the polarization consistent with the orbital period. 
The intrinsic polarization in X-ray binary systems can result from nonthermal emission processes due to the presence of high-energy electrons in the relativistic jets or, alternatively, by electron Thomson scattering due to an extended plasma above an accretion disk or a stellar envelope. Moreover, this intrinsic polarization could change with the binary phase, due to the polarizing mechanism, to the distribution and physical state of the material, or by geometric factors such as the inclination angle.

The main problem in the effective identification of intrinsic polarization of a source at optical frequencies is the contamination produced by the Galactic interstellar medium (ISM) polarization. This component is thought to arise due to extinction by anisotropic dust particles preferentially oriented by the Galactic magnetic field. At present, the most complete study of Galactic starlight polarization has been carried out by Fosalba et al. (2002). They presented a statistical analysis of the Galactic ISM polarization using $\sim 5500$ stars and found that the interstellar grains are not fully aligned with the Galactic magnetic field, a fact that was interpreted as the effect of a large random component of the field. However, it is important to stress that an entire reconstruction of the three-dimensional magnetic orientations requires additional radio, submillimeter and infrared data.

In this paper, we report the first optical polarimetry of LS 5039. We describe the observations in Sect. 2, present the obtained results in Sect. 3, and we discuss on the origin of the detected polarization and state our conclusions in Sect. 4.

\section{Optical observations}

\subsection{Polarimetry}

Polarimetric observations of LS 5039 were obtained at the $2.15 \mathrm{~m}$ "Jorge Sahade" telescope, CASLEO, Argentina, using a two-channel polarimeter. This instrument is a rotating plate polarimeter with a Wollaston prism that splits the incident light beam into two components, each one directed to a different photomultiplier (Martínez et al. 1990). Its optical design is based on VATPOL, the Vatican Observatory polarimeter (Magalhães et al. 1984).

The observations spanned four consecutive nights, roughly centered at the new moon, from 2003 June 28 to July 1 (UT dates). Weather conditions were fairly good for most of the observing time, except for thick cirrus during the second night and thin cirrus during a few hours at the beginning of the first and third nights. The target was repeatedly observed through $V$ and $I$ filters, closely matching the Johnson-Cousins system, with individual exposure times of 300-360 s; this yielded between 8 and 12 individual data points each night in each band. Sky integrations were regularly obtained between science exposures. A 11.3" diaphragm was used for all the observations.

Six field stars, with apparent magnitudes bracketing that of LS 5039 and within $\sim 3.5^{\prime}$ from its position (see Fig. 1), were also observed in order to estimate the foreground polarization. Both polarized and unpolarized stars from the lists of Turnshek et al. (1990) were also observed each night, in order to evaluate the instrumental polarization (which was found to be practically zero) and to correct the measured polarization angles to

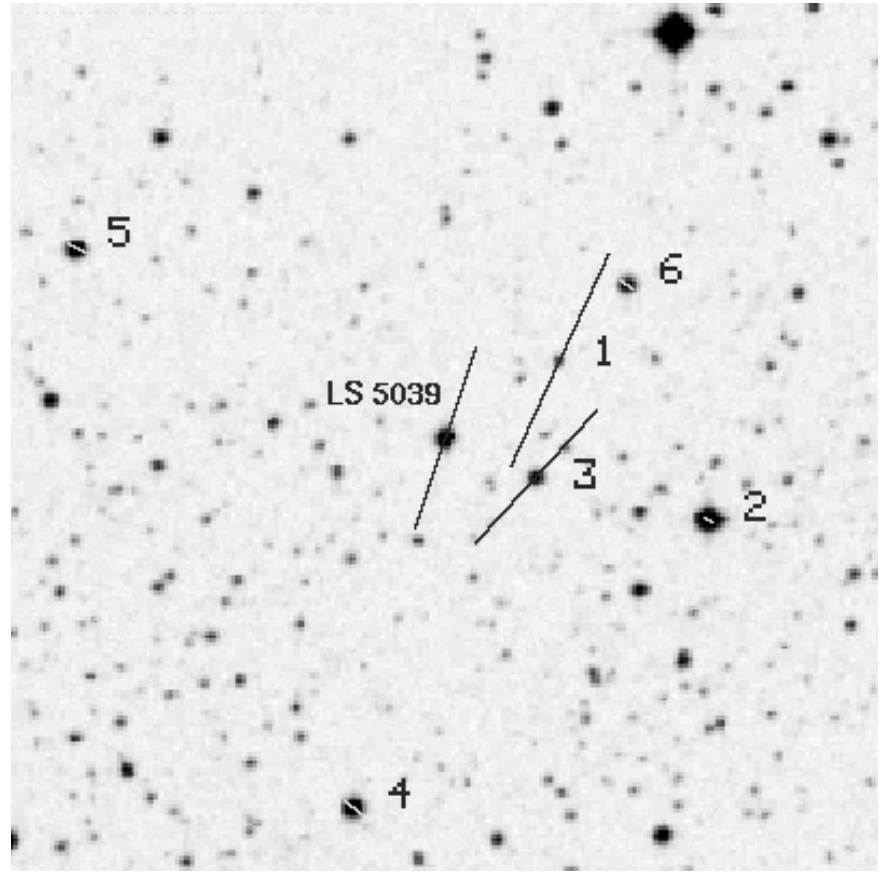

Fig. 1. DSS 7" $\times 7 "$ image centered on LS 5039, showing the target and the six comparison stars. Polarization vectors in the $V$ band have been overlaid. North is up and East is to the left.

the equatorial standard system. We were able to determine this zero point correction to an accuracy of $\pm 5^{\circ}$.

Since both the ordinary and the extraordinary rays are measured almost simultaneously, polarimetric observations should be barely affected by non-photometric conditions. However, large and/or rapid variations in the sky polarization (e.g., when the Moon is rising or setting) are expected to introduce systematic errors in the measured polarization, because the sky conditions may have changed between the object and sky measurements. To prevent against this source of error, we interpolated the sky brightness and polarization (in the Stokes $U-Q$ plane) to the same instant of each object observation.

As a further check, we plotted the magnitudes and Stokes parameters of both object and sky as a function of time, in order to verify whether or not any change in the observed target polarization may have been caused by a sudden change in the sky polarization and/or transparency. This allowed us to reject some suspicious data points, as will be explained below.

\subsection{Spectroscopy}

Spectroscopic observations for the most polarized ( 5\%) comparison stars were conducted on 2003 October 13 with the $2.56 \mathrm{~m}$ Nordic Optical Telescope (NOT) in the island of La Palma (Spain). The instrument used was the Andalucia Faint Object Spectrograph and Camera (ALFOSC) combined with Grism 6 and the CCD detector EEV42-40 $(2 \mathrm{~K} \times 2 \mathrm{~K})$. The CCD frames were processed using standard tasks in the IRAF package, including bias subtraction, flat-fielding, optimal extraction and wavelength calibration based on arc frames taken with a helium lamp. 
Table 1. Average polarization and position angle measurements of the comparison stars and LS 5039.

\begin{tabular}{ccrcr}
\hline \hline $\begin{array}{c}\text { Object Name } \\
\text { USNO-A2.0 0750- }\end{array}$ & $\begin{array}{c}\left\langle P_{V}\right\rangle \\
(\%)\end{array}$ & \multicolumn{1}{c}{$\begin{array}{c}\left\langle\theta_{V}\right\rangle \\
\left.{ }^{\circ}\right)\end{array}$} & \multicolumn{1}{c}{$\left\langle P_{I}\right\rangle$} & \multicolumn{1}{c}{$\begin{array}{c}\left\langle\theta_{I}\right\rangle \\
(\%)\end{array}$} \\
\hline $13157416(\# 1)$ & $6.36 \pm 0.41$ & $155.2 \pm 1.8$ & $4.75 \pm 0.11$ & $155.9 \pm 0.7$ \\
$13153701(\# 2)$ & $0.32 \pm 0.04$ & $67.7 \pm 3.7$ & $0.42 \pm 0.01$ & $58.5 \pm 1.0$ \\
$13157934(\# 3)$ & $4.92 \pm 1.00$ & $137.5 \pm 5.8$ & $5.67 \pm 0.08$ & $162.3 \pm 0.4$ \\
$13162766(\# 4)$ & $0.58 \pm 0.02$ & $54.2 \pm 1.2$ & $0.45 \pm 0.02$ & $48.7 \pm 1.3$ \\
$13170022(\# 5)$ & $0.51 \pm 0.04$ & $65.2 \pm 2.0$ & $0.47 \pm 0.03$ & $57.2 \pm 1.7$ \\
$13155723(\# 6)$ & $0.47 \pm 0.07$ & $54.8 \pm 4.3$ & $0.40 \pm 0.06$ & $58.1 \pm 3.9$ \\
\hline LS 5039 & $5.26 \pm 0.15$ & $161.3 \pm 0.8$ & $4.70 \pm 0.16$ & $162.4 \pm 1.0$ \\
\hline
\end{tabular}

\section{Results}

The polarization and position angle measurements in the $V$ and $I$ bands of the six comparison stars, and average values for LS 5039, are quoted in Table 1. We show in Fig. 1 a Digitized Sky Survey (DSS) image of the target field with polarization vectors in the $V$ band overlaid onto LS 5039 and the six comparison stars.

It is evident that, at least, two different polarization patterns are produced by interstellar dust along the studied direction. Stars \#2, \#4, \#5, and \#6 show polarization vectors with small amplitudes $\left(\left\langle P_{V}\right\rangle \sim 0.3-0.6 \%\right)$, roughly aligned in the NE-SW direction $\left(\left\langle\theta_{V}\right\rangle \sim 60^{\circ}\right)$. On the other hand, light from stars \#1 and \#3 is polarized almost at an orthogonal direction $\left(\left\langle\theta_{V}\right\rangle \sim 135-155^{\circ}\right)$, with a significantly higher percentage $\left(\left\langle P_{V}\right\rangle \sim 5-6 \%\right)$; hence, these two stars have polarization properties very similar to that of LS 5039.

We show in Fig. 2 the polarization measurements of LS 5039 in the $V$ (filled circles) and $I$ (open circles) bands as function of the orbital phase $\left(t_{0}=\right.$ HJD $2452756.49, P_{\text {orb }}=$ $4.4267 \mathrm{~d}$ ). During the first night, corresponding to $\Phi \simeq 0.05$ in Fig. 2, the sky polarization remained fairly constant at $\sim 1.5 \%$, both in $V$ and $I$. Since, in addition, its flux contribution within the diaphragm was $4.5-5$ mag lower than that from LS 5039, no significant systematic errors from sky polarization are expected. The $I$ band polarization shows no significant variations with time, remaining nearly constant at $\left\langle P_{I}\right\rangle=4.6 \%$. In turn, the $V$ band polarization time-curve shows fluctuations at a $\$ 3 \sigma$ level, except for two points falling significantly below the mean. These data correspond to brightenings in the lightcurve, and the larger variation in $P_{V}$ was accompanied by a $\sim 4^{\circ}$ change in $\theta_{V}$. Although instrumental errors as the sources for these variations cannot be absolutely ruled out, there is no indication that any such error could have induced the observed behaviour. However, this would contrast with the apparent photometric stability of LS 5039 within few hundredths of magnitude reported by several authors (e.g. Martí et al. 2004).

On the second night ( $\Phi \simeq 0.30$ ), as stated above, we observed through thick cirrus. As a consequence, the quality of these data is not as good, with large fluctuations (up to $\sim 4 \%$ ) in the sky polarization; hence, significant systematic errors could be affecting this data set. The first half of the third night $(\Phi \simeq 0.50)$ was affected by thin cirrus, so we had to discard a few suspicious data points. Finally, on the fourth night
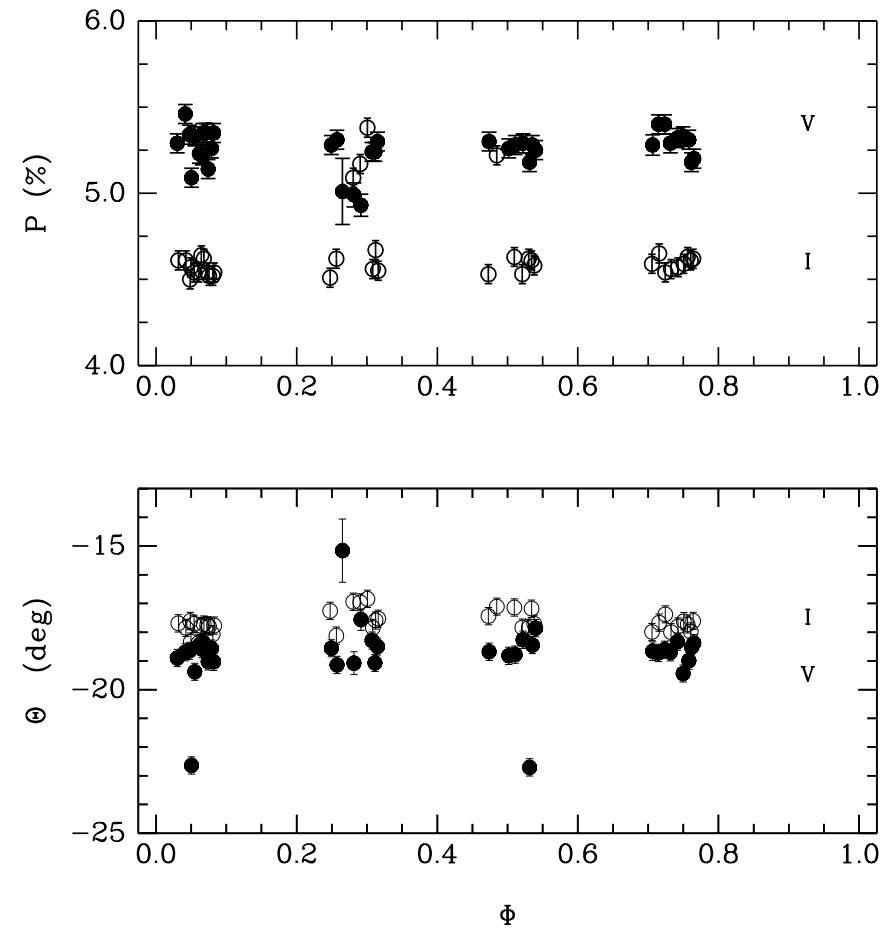

Fig. 2. Polarization and position angle in the $V$ (filled circles) and $I$ (open circles) bands as a function of the orbital phase for LS 5039.

$(\Phi \simeq 0.75)$ we observed mostly under photometric conditions. The object was constantly $\sim 4$ mag brighter than the sky within the diaphragm, and no rapid changes in the sky polarization were evident. No significant systematic errors should therefore be expected.

Concerning the NOT spectroscopic observations, the sky conditions were non-photometric and affected by clouds. Moreover, we could only acquire spectra with the objects being at a significant air mass. Consequently, a spectrum suitable for classification was only obtained for the brightest comparison star \#3. After combining two 600 s exposures, the resulting spectrum is consistent with an A0 Ib star taking into account the strong narrow hydrogen absorption lines.

On the other hand, the apparent magnitudes of star \#3 are $m_{B}=13.92 \pm 0.04$ and $m_{V}=12.86 \pm 0.04$, hence $B-V=$ $1.06 \pm 0.06$ (Martí et al. 2004). For an A0 supergiant, the absolute magnitude is $M_{V}=-5.3 \pm 0.1$ and the intrinsic color $(B-V)_{0}=0.00$ (Allen 1973). Therefore, this comparison star has a color excess of $E(B-V)=1.06 \pm 0.06 \mathrm{mag}$. Using the relationship $A_{V}=\left(3.30+0.28(B-V)_{0}+0.04 E_{B-V}\right) E(B-V)$ (Schmidt-Kaler 1982), this corresponds to an interstellar extinction of $A_{V}=3.5 \pm 0.2$ and a distance of $8.4 \pm 0.1 \mathrm{kpc}$.

\section{Discussion}

The observed polarization in astrophysical objects is usually the sum of two components, the intrinsic one produced in the object and the one arising from the foreground ISM between the object and the observer. The high polarization measured towards LS 5039 ( $\sim 5 \%$ in $V$ band) suggests that, at least, part of it should have an intrinsic origin because it is very unusual to be produced in the ISM up to a distance of $3 \mathrm{kpc}$. 
To deduce the intrinsic polarization of the source, $P_{\mathrm{IN}}$, it is necessary to know the contribution of the ISM polarization, i.e. the value of $P_{\text {ISM }}$ and subtract it from the observed one.

First of all, we assume that light emitted from stars \#1 to \#6 is not polarized. The similar percentages and angles of polarization of stars \#2, \#4, \#5 and \#6, suggest that they are being slightly affected by the ISM and that they lie close to the Sun, possibly in the local spiral arm (distance $<500 \mathrm{pc}$ ). On the contrary, stars \#1 and 3\# have polarization properties similar to that of LS 5039.

As we have mentioned in previous sections, the main problem to determine the intrinsic polarization of an object, is the absence of a complete study on the Galactic interstellar polarization. However, we can obtain a rather crude estimation of the intrinsic polarization of LS 5039 by combining the statistical study of galactic interstellar polarization carried out by Fosalba et al. (2002) and all the information gathered by us from polarimetric and spectroscopic observations of the other stars in the field.

Fosalba et al. (2002), have found that the distribution of the polarization degree as a function of distance grows linearly up to $\sim 2 \mathrm{kpc}$, but beyond this distance the behavior of $P(\%)$ is best fitted by third-order polynomials up to a distance of $\sim 6 \mathrm{kpc}$ (see their Fig. 3). Adopting a distance of $\sim 2.9 \mathrm{kpc}$ for LS 5039 (Ribó et al. 2002) the polarization percentage as a function of the distance results $\sim 2.3 \%$. From the equations that give the behavior of the polarization degree with Galactic longitude and latitude, we obtain values for $P(\%) \sim 0.8$ and $P(\%) \sim 2$, respectively. On the other hand, if we assume that the contribution of the interstellar polarization increases linearly with distance, we derive $P_{\text {ISM }} \sim 2.5 \%$ a value consistent with those obtained from Fosalba et al. (2002), for the set of known stellar parameters of LS 5039. So, we suggest that the intrinsic polarization of LS 5039 is $~ 3 \%$.

This value is also similar to the one obtained by Gliozzi et al. (1998) for the microquasar GRO J1655-40, which was interpreted as the consequence of electron scattering by plasma above the accretion disk.

The origin of the intrinsic polarized optical emission estimated for LS 5039 could be due to a nonthermal emission process or to electron Thomson scattering. If the observed polarized flux is the result of synchrotron emission originated from the relativistic plasma inside the jet, then the radio electron populations should play an important role in the generation of optical emission. However, we have extrapolated the energy distribution of the jet in LS 5039 (Ribó 2002) from radio to optical frequencies and find that it is at least 4 orders of magnitude lower than the observed flux. Since the nonthermal emission process is not enough to generate the polarized optical emission detected towards LS 5039, the electron Thomson scattering appears as a suitable alternative mechanism causing the observed polarized flux. Since there is no clear evidence of the presence of a large accretion disk in LS 5039, the most likely possibility is that the polarized emission originates in the stellar envelope of the companion star. Such scenario has been previously proposed by Brown \& McLean (1977) to explain the intrinsic polarization by Thomson scattering on free electrons in optically thin stellar envelopes. An improved treatment is given in Brown et al. (1978), while other works on stellar scattering polarization can be found in Cassinelli et al. (1987) and references therein.

Considering the Brown \& McLean picture for the generation of intrinsic polarization, we can estimate some physical and geometrical parameters for LS 5039. Assuming that the plasma fills an ellipsoidal shell of any thickness with a uniform density distribution $n_{0}$ of scattering electrons, the expected polarization is given by:

$P=\sigma_{\mathrm{T}} n_{0} R(1-3 \gamma) \sin ^{2} i$

where, $\sigma_{\mathrm{T}}$ is the Thomson cross-section, $R$ is the radius of the envelope, $i$ is the inclination angle of the equatorial plane and $\gamma$ is a function of the ratio $a$ of the equatorial to polar radius. Assuming a simple radial flow, the corresponding electron density of the envelope is:

$$
n_{0} \sim \frac{\dot{M}_{\mathrm{opt}}}{4 \pi R^{2} v_{\infty}\left[1-\frac{R_{\mathrm{opt}}}{R}\right]^{\beta} \mu m_{\mathrm{H}}} .
$$

Following McSwain et al. (2004), suitable values for LS 5039 are a wind velocity at infinity $v_{\infty}=2440 \pm 190 \mathrm{~km} \mathrm{~s}^{-1}, \beta=1.0$, an optical star radius $R_{\text {opt }}=8.5 R_{\odot}$ and a wind mass loss $\dot{M}_{\text {opt }}=4 \times 10^{-8} M_{\odot} \mathrm{yr}^{-1}$ (the last two quantities are mean values). The other symbols have their usual meanings. Introducing Eq. (1) into Eq. (2) and considering values of $\gamma$ of $0.1,0.2$ and $0.3(P(\%)>0)$ we estimate that the density of the scattering electrons is in the range $(0.2-1.3) \times 10^{12} \mathrm{~cm}^{-3}$. From the above results we conclude that the polarized emission originates mainly close to the star at a distance between (8.51-8.6) $R_{\odot}$, where the velocity of the stellar wind is $(3-20) \mathrm{km} \mathrm{s}^{-1}$.

An interesting feature in the polarization of LS 5039 is the detected variability during the first and second nights. If the polarimetric fluctuations observed on timescales of hours are real, then they might be attributed to localized density enhancements (or blobs) propagating in the general wind. The existence of these blobs has been introduced by Brown et al. (1995) to explain transient features seen in polarimetric light curves of WR stars. Further simultaneous photometric and polarimetric observations of the system are necessary to shed light on the origin of the variable optical polarization.

Acknowledgements. We acknowledge Pablo Fosalba for useful discussions. J.A.C. is a researcher of the programme Ramón y Cajal funded jointly by the Spanish Ministerio de Ciencia y Tecnología and Universidad de Jaén, and was also supported during this work by CONICET (under grant PEI 6384/03). J.A.C. is very grateful to staff of the Service d'Astrophysique (CEA Saclay) where part of this research was carried out. J.M. and M.R. acknowledge partial support by DGI of the Ministerio de Ciencia y Tecnología (Spain) under grant AYA2001-3092, as well as partial support by the European Regional Development Fund (ERDF/FEDER). J.M. is also supported by the Junta de Andalucía (Spain) under project FQM322. M.R. acknowledges support by a Marie Curie Fellowship of the European Community programme Improving Human Potential under contract number HPMF-CT-2002-02053. The Digitized Sky Survey was produced at the Space Telescope Science Institute under US Government grant NAG W-2166. 


\section{References}

Allen, C. W. 1973, Astrophysical Quantities (London: The Athlone Press Ltd.)

Brown, J. C., \& McLean, I. S. 1977, A\&A, 57, 141

Brown, J. C., McLean, I. S., \& Emslie, A. G. 1978, A\&A, 68, 415

Brown, J. C., Richardson, L. L., Antokhin, I., et al. 1995, A\&A, 295, 725

Cassinelli, J. P., Nordsieck, K. H., \& Murison, M. A. 1987, ApJ, 317, 290

Fosalba, P., Lazarian, A., Prunet, S., \& Tauber, J. A. 2002, ApJ, 564, 762

Gliozzi, M., Bodo, G., Ghisellini, G., Scaltriti, F., \& Trussoni, E. 1998, A\&A, 337, L39

Hartman, R. C., Bertsch, D. L., Bloom, S. D., et al. 1999, ApJS, 123, 79

Magalhães, A. M., Benedetti, E., \& Roland, E. H. 1984, PASP, 96, 383

Martí, J., Paredes, J. M., \& Ribó, M. 1998, A\&A, 338, L71

Martí, J., Luque-Escamilla, P., Garrido, J. L., Paredes, J. M., \& Zamanov, R. 2004, A\&A, 418, 271
Martínez, E., Aballay, J. L., Marún, A., \& Ruartes, H. 1990, Bol. Asoc. Arg. de Astronomía, 36, 342

McSwain, M. V., \& Gies, D. R. 2002, ApJ, 568, L27

McSwain, M. V., Gies, D. R., Huang, W., et al. 2004, ApJ, 600, 927

Paredes, J. M., Martí, J., Ribó, M., \& Massi, M. 2000, Science, 288, 2340

Paredes, J. M., Ribó, M., Ros, E., Martí, J., \& Massi, M. 2002, A\&A, 393, L99

Reig, P., Ribó, M., Paredes, J. M., \& Martí, J. 2003, A\&A, 405, 285

Ribó, M. 2002, Ph.D. Thesis, Universitat de Barcelona

Ribó, M., Reig, P., Martí, J., \& Paredes, J. M. 1999, A\&A, 347, 518

Ribó, M., Paredes, J. M., Romero, G. E., et al. 2002, A\&A, 384, 954

Scaltriti, F., Ghisellini, G., Gliozzi, M., \& Trussoni, E. 1997, A\&A, 325, L29

Schmidt-Kaler, Th. 1982, Landolt-Börnstein: Numerical Data and Functional Relationships in Science and Technology, New Series, Group 6 Astronomy and Astrophysics, Vol. 2

Turnshek, D. A., Bohlin, R. C., Williamson, R. L., et al. 1990, AJ, 99, 1243 